\title{
Response programming vs. alternative interpretations of the "dit-dah" reaction time effect
}

\author{
STUART T. KLAPP, JoELLEN McRAE, and WILLIAM LONG \\ California State University, Hayward, California 94542
}

\begin{abstract}
In a three-choice reaction time paradigm, subjects made buttonpress responses of short, medium, or long duration. Reaction time increased monotonically with the three levels of required response duration for each subject. This result is difficult to accommodate with a hypothesis involving anticipation of stimulus events, and is consistent with the previous view that the difference in reaction time reflects differences in the times required to program the subsequent responses. Within each condition of required press duration, the within-subject correlation of reaction time and actual response duration was essentially zero. This result is consistent with the programming interpretation of the above between-conditions effect rather than an alternative interpretation of a general slowing of the time frame of the reaction time/ response duration sequence.
\end{abstract}

Previous experiments in which the first author has been involved examined reaction time prior to the beginning of a buttonpress response as a function of the required duration of the press, that is, whether a short "dit" or longer "dah" press duration was to be generated (Klapp, in press-a; Klapp \& Wyatt, 1976; Klapp, Wyatt, \& Lingo, 1974). Choice reaction time was longer prior to the longer press ("dah") than prior to the shorter press ("dit") when measured in a twochoice paradigm. By contrast, for the simple reaction time paradigm, in which the response duration was cued in advance of the reaction time interval, this effect did not appear for motivated subjects, who were assumed to use the advance cue. The difference in choice reaction times was interpreted as representing the difference in programming times for generating the timing of the "dit" and "dah" responses (Klapp, in press-b). It is further assumed that programming can occur in advance and the resultant program stored until needed for the simple reaction time case (Klapp, 1976). This explains why the difference in reaction time does not appear for simple reaction time.

The purpose of the present study was to consider two alternative interpretations of the finding that choice reaction time depends on the targeted response duration. According to the first alternative, subjects tend to anticipate or predict the occurrence of the signal calling for a "dit." Reaction time is shorter when the actual signal agrees with the anticipation

This research was supported in part by Grant BNS 76-13466 from the National Science Foundation. The authors are indebted to Gregory Messer, who designed and constructed the apparatus. Requests for reprints may be addressed to Stuart T. Klapp, Department of Psychology, California State University, Hayward, California 94542. than when it does not, thereby producing the shorter reaction time prior to "dit" compared to "dah." This same alternative interpretation also handles the result that simple reaction time is constant across "dit" and "dah" responses since subjects would not falsely anticipate after the correct response had been cued. In the present experiment the two-choice "dit" vs. "dah" paradigm was replaced with a three-choice paradigm involving short, medium, or long presses. According to the programming hypothesis, reaction time should increase monotonically as a function of required response duration. By contrast, it is difficult to handle a monotonic function with the anticipation hypothesis. For example, if subjects tend to anticipate one of the responses, reaction time for that alternative will be short compared to the others, yielding a nonmonotonic relation between response duration and reaction time.

According to the second alternative interpretation to be considered, the relation between reaction time and targeted response duration may be due to a general slowing of the time frame of the reaction time/response sequence rather than to a preparatory process. According to this alternative, reaction time should be related to produced response duration, as well as to the targeted response duration. Therefore, reaction time should depend on duration within the same condition of targeted response duration. If, on the other hand, the effect of targeted duration on reaction time is due to programming, then no such relation should be observed, since the same programming process applies to all responses within a targeted condition. These possibilities were tested in the present experiment by computing the correlation between reaction time and response duration within condition and within subject. 


\section{METHOD}

The response was to press a button which sounded a tone. The duration of the press was to be short $(150 \pm 50 \mathrm{msec})$, medium $(300 \pm 100 \mathrm{msec})$, or long $(600 \pm 200 \mathrm{msec})$. The tolerance became broader as the response duration increased so that the tolerance band was a constant proportion of the targeted duration, with a broader absolute tolerance for the longer responses. This arrangement avoided having tighter absolute or relative tolerances for the longer responses for which a longer reaction time was anticipated. Therefore, any increase in reaction time with response duration could not be due to confounded accuracy requirements. Responses for which the duration was within tolerance were signaled with a feedback tone. Incorrect responses were signaled by one of two red lights indicating that the duration had been too long or two short. The error trials were repeated and only the data from correct responses were included in the analysis.

Each trial was started with the display of a warning signal for $500 \mathrm{msec}$. Then, after a randomly determined foreperiod of 1,300 or $1,500 \mathrm{msec}$, one of three signal lamps was displayed indicating whether the response duration was to be short, medium, or long. The three signal lamps were placed in a horizontal row, subtending a visual angle of $4.2 \mathrm{deg}$. For half of the subjects the lights were short, medium, and long, reading left to right; this order was reversed for the remaining subjects. Reaction time was measured from the onset of one of the lights until the button was pressed. The order in which the short, medium, and long response signals appeared was randomized independently for each subject in blocks of 24 trials, with 8 trials per response in each block. Three such blocks ( 72 trials) were given to each subject.

The eight subjects were students in introductory psychology at California State University, Hayward, who participated in order to fulfill a course requirement. For each subject the mean reaction time was computed for the 24 trials of each required response duration, and the correlation between reaction time and actual response duration was computed for trials within these sets of 24 trials.

\section{RESULTS AND DISCUSSION}

The rate of incorrect responses increased as a function of required response duration: $12 \%, 17 \%$, and $18 \%$ for the short, medium, and long responses, respectively. Therefore, any increase in reaction time with response duration could not be attributed to speed-accuracy tradeoff.

The mean reaction time increased as a function of required buttonpress duration, 335,394 , and $413 \mathrm{msec}$ $[\mathrm{F}(2,14)=17.1, \mathrm{p}<.001]$. This monotonic relation appeared individually for each of the eight subjects ( $p<.01$, sign test, for both the short vs. medium and medium vs. long comparisons). This result is difficult to explain by the anticipation hypothesis. In order to hold such an interpretation, one would have to assume that each subject distributes his anticipations among the three responses such that the probability of anticipation is inversely related to the required duration. The simple notion that one alternative is anticipated cannot handle the data.

For each set of 24 trials within each targeted duration condition for each subject, the correlation between reaction time and actual duration was computed. These correlations ranged from +.51 to -.52 , with a mean of -.06 . Of the 24 correlations, only 6 tested as significant at the $p<.05$ level, and 4 of the 6 were negative. We conclude that there is little evidence of any positive relation between reaction time and actual response duration within condition. This result is not consistent with the view that the relation between targeted response duration and reaction time, observed between conditions, is due to a general slowing of all phases of the response.

Having rejected both the anticipation and the general slowing alternative interpretations, we conclude that it is reasonable to continue to interpret the effect of required press duration on choice reaction time in programming terms.

\section{REFERENCES}

KLAPP, S. T. Short-term memory as a response preparation state. Memory \& Cognition, 1976, 4. 721-729.

KLAPP. S. T. Rèsponse programming, as assessed by reaction time, does not establish commands for particular muscles. Journal of Motor Behavior, in press. (a)

KLAPP. S. T. Reaction time analysis of programmed control. In R. Hutton (Ed.), Exercise and sports sciences review's. Santa Barbara, Calif: Journal Publishing Affiliates, in press. (b)

KLAPP, S. T., \& Wyatt, E. P. Motor programming within a sequence of responses. Journal of Motor Behavior, 1976. 8. 19-26.

Klapp, S. T., Wyatt, E. P., \& Lingo, W. M. Response programming in simple and choice reactions. Journal of Motor Behavior, 1974, 6, 263-271.

\section{NOTE}

1. Fortunately, most other examples of the "programming effect" from our laboratory are not open to this alternative interpretation (see Klapp, in press-b).

(Received for publication September 19, 1977.) 